\title{
New directions in neuroprotection: Basic mechanisms, molecular targets and treatment strategies
}

\author{
L.M. Refolo and H.M. Fillit
}

Few diseases are as devastating as the neurodegenerative ones including: Alzheimer's disease (AD), Parkinson's disease (PD) and Amyotrophic Lateral Sclerosis (ALS), to name a few. Genetic risk-factors influence these age-associated, chronic illness but, by and large, the causes of these diseases remain unknown. There are known familial forms of AD, ALS and PD and the identification of mutations in specific genes causing each of these degenerative disorders has provided opportunities to investigate the molecular participants in the disease process and pathogenic mechanisms. However, the familial forms of these diseases account for only a small percentage of observed cases; the sporadic forms of the diseases are either caused by undiscovered genes or, molecular events and multifactorial risk factors and pathways that we do not completely understand.

The neurodegenerative diseases are generally characterized by the dysfunction and death of specific populations of neurons and in many cases by the presence, in brain, of intracellular or extracellular aggregates of misfolded protein(s). Neuronal death occurs either chronically, such as in Alzheimer's disease, which involves neuronal loss over years to decades or, acutely within days, such as in, head trauma, stroke and spinal cord injury. Broadly speaking there are three types of neuronal death: necrosis which can trigger neuroinflammation and involves secondary damage to healthy neighboring neurons, apoptosis which is non-inflammatory and is less damaging to neighboring neurons and, paraotosis which represents an intermediate between apopotosis and necrosis. Each type of cell death may have a unique set of pathways and mechanisms leading to neuronal death. However, many of the underlying neurotoxic mechanisms are shared across a wide variety of neurodegenerative diseases (e.g. $\mathrm{Ca}^{2+}$ excess, generation of reactive oxygen species, chronic inflammation, caspase activation).

The development of therapies for the neurodegenerative disease represents a major challenge to academic, biotechnology and pharmaceutical scientists. Current therapies for the neurodegenerative diseases provide effective symptomatic relief, particularly in early stages of disease (e.g., memantine and cholinesterase inhibitors for AD, dopaminergic drugs for PD, Rilutek for ALS). However, there are too few, if any therapies that affect the underlying disease processes. Therefore, disease- modifying therapies that halt, slow or reverse disease progression are sorely needed. It is expected that, next generation of neuroprotective therapies will stop and/or slow neuronal cell death and will therefore be disease modifying.

Using the tools of modern neuroscience, neurology, pharmacology, chemistry, genomics, proteomics, psychometrics and bioinformatics, neuroscientists have made good progress in not only in understanding some of the mechanisms of neurodegeneration but, also in identifying and validating new drug targets which are the basis for novel therapeutic strategies. For example, a number the molecular and cellular factors that trigger the different types of neuronal cell death have been revealed; represent targets for neuroprotective strategies. Cellular factors include oligodendrocytes, activated microglia and astroglia. These generate molec- 
ular factors such as pro-inflammatory cytokines, e.g., the tumor necrosis factor family, interleukin family, adhesion receptors (VCAM) and intercellular adhesion molecules (ICAM) that have a major role in neuronal apoptosis. Molecular mechanisms subserving neuronal cell death pathways include: over activation of NMDA receptors, accumulation of excess post-syna ptic $\mathrm{Ca}^{2+}$, activation of Jun $\mathrm{N}$-terminal kinase (JNK), activation of cell death receptors (e.g. Fas/CD95 and tumor necrosis factor receptor), mitochondrial dysfunction (release of cytochrome c), caspase activation, generation of reactive oxygen species (ROS) and accumulation of misfolded proteins, to name a few. Each of these mechanisms is associated with a number of genes and gene products that provide targets for drug discovery and strategies for therapeutic intervention.

Advances in understanding the molecular mechanisms of neurodegeneration have revealed therapeutic strategies that are now in the process of development. These include: neuroprotective gene therapy (e.g. neurotrophins NGF and BDNF, over expression of SOD), small molecule neurotrophin mimetics, small molecule caspase inhibitors, inhibitors of cell-death promoting transcription factors, molecules which stimulate neuronal regeneration (e.g. neurogenic compounds), kinases inhibitors (JNK and MAP), molecules that block glutamate -induced $\mathrm{Ca}^{2+}$ influx (e.g. memantine and other NMDA receptor channel blockers).

Continued efforts to understand the regulation of neurodegeneration and the functions of individual molecules comprising the cell death machinery should reveal additional strategies for preventing, halting and reversing neuronal cell loss. The coming decade represents an important and exciting period for drug discovery for neurodegnerative disease, as these new therapies begin to reach the clinic for assessment in patients.

To address issues in developing new neuroprotective therapies ISOA convened a "catalyst conference" to advance drug discovery for neurodegenerative diseases.

The objectives of this multidisciplinary conference are to:

- Discuss basic mechanisms of neuronal injury and cell death.

- Review the pathways leading to neuronal cell death and identify potential molecular targets for intervention.

- Review pathways that enhance cell survival.

- Identify new areas of neurobiological research that will lead to a more complete understanding of the cell death machinery and reveal novel therapeutic targets.

- Discuss and evaluate current neuroprotective agents, strategies and clinical trials.

- Focus on developing the next generation of neuroprotective agents and innovative human neuroprotection trials.

The conference was supported by a kind gift from Forest Laboratories, Inc. 